\title{
EMBEDDING OF COMPLETE MULTIPARTITE GRAPHS INTO CYCLE-OF-LADDERS
}

\author{
Jia-Bao Liu ${ }^{1}$, R. Karthik ${ }^{2} \S$ \\ S. Rethina Kumar ${ }^{3}$ \\ ${ }^{1}$ School of Mathematics and Physics \\ Anhui Jianzhu University, Hefei 230601, P.R. CHINA \\ ${ }^{2}$ Department of Mathematics \\ Velammal Engineering College, Chennai, INDIA \\ ${ }^{3}$ Department of Mathematics \\ Thanthai Hans Roever College, Perambalur, INDIA
}

\begin{abstract}
Graph embedding is the mapping of a topological structure (guest graph) into another topological structure (host graph) that preserves certain required topological properties and the graph embedding ability reflects how efficiently a parallel algorithm with a guest graph can be executed on a host graph [1] and the utilization of system resources in the host graph [2]. In this paper, we obtain exact wirelength of embedding complete multipartite graphs into cycle-of-ladders.
\end{abstract}

AMS Subject Classification: 05C07, 05C35, 05C40

Key Words: embedding; congestion; wirelength; complete multipartite graphs; cycle-of-ladders

\section{Introduction}

Recent advances in integrated circuit technology make it possible to construct very large interconnection networks. Together with these advances, many interconnection network topologies have been proposed and investigated in the literature. Interconnection networks are often modeled as graphs. Graph embedding is an important factor to evaluate an interconnection network. Em-

Received: June 17, 2019

(C) 2019 Academic Publications

${ }^{\S}$ Correspondence author 
bedding as mathematical models of parallel computing have been discussed extensively in the literature. In this model, both the algorithm to be implemented and the interconnection network of the parallel computing system are represented by graph [3], the implementation details are then studied through the embedding.

Let $G(V, E)$ and $H(V, E)$ be finite grahs with $n$ vertices. An embedding $f$ of $G$ into $H$ is defined [4] as follows:

1. $f$ is a bijective map from $V(G) \rightarrow V(H)$,

2. $f$ is a one-to-one map from $E(G)$ to $\left\{P_{f}(u, v): P_{f}(u, v)\right.$ is a path in $H$ between $f(u)$ and $f(v)$ for $(u, v) \in E(G)\}$.

The edge congestion of an embedding $f$ of $G$ into $H$ is the maximum number of edges of the graph $G$ that are embedded on any single edge of $H$. Let $E C_{f}(G, H(e))$ denote the number of edges $(u, v)$ of $G$ such that $e$ is in the path $P_{f}(u, v)$ between $f(u)$ and $f(v)$ in $H$. In other words,

$$
E C_{f}(G, H(e))=\left|\left\{(u, v) \in E(G): e \in P_{f}(u, v)\right\}\right|,
$$

where $P_{f}(u, v)$ denotes the path between $f(u)$ and $f(v)$ in $H$ with respect to $f$. If we think of $G$ as representing the wiring diagram of an electronic circuit, with the vertices representing components and the edges representing wires connecting them, then the edge congestion $\operatorname{EC}(G, H)$ is the minimum, over all embeddings $f: V(G) \rightarrow V(H)$, of the maximum number of wires that cross any edge of $H$, [5]. Other undefined notation and terminology are in $[6,7,8,9]$.

The Wirelength Problem. The wirelength of an embedding $f$ of $G$ into $H$ is given by

$$
W L_{f}(G, H)=\sum_{(u, v) \in E(G)} d_{H}(f(u), f(v))=\sum_{e \in E(H)} E C_{f}(G, H(e)),
$$

where $d_{H}(f(u), f(v))$ denotes the length of the path $P_{f}(u, v)$ in $H$. Then, the wirelength of $G$ into $H$ is defined as

$$
W L(G, H)=\min W L_{f}(G, H),
$$

where the minimum is taken over all embeddings $f$ of $G$ into $H$. The wirelength problem $[10,11,12]$, of a graph $G$ into $H$ is to find an embedding of $G$ into $H$ that induces the minimum wirelength $W L(G, H)$. 
The wirelength of a graph embedding arises from VLSI designs, data structures and data representations, networks for parallel computer systems, biological models that deal with cloning and visual stimuli, parallel architecture, structural engineering and so on[13]. Graph embeddings have been well studied for meshes into crossed cubes [14], binary trees into hypercubes [15], complete binary trees into hypercubes [16], incomplete hypercube in books [17], tori and grids into twisted cubes [18], meshes into locally twisted cubes [19], generalized ladders into hypercubes [20], grids into grids [21], binary trees into grids [22], hypercubes into cycles [23], star graph into path [24], meshes into M obius cubes [25], ternary tree into hypercube [26], enhanced and augmented hypercube into complete binary tree [27], circulant into arbitrary trees, cycles, certain multicyclic graphs and ladders [28], hypercubes into cylinders, snakes and caterpillars [29], embedding of special classes of circulant networks, hypercubes and generalized Petersen graphs [30].

In this paper, we present an algorithm for finding the embedding of Complete multipartite graphs into cycle-of-ladders and prove its correctness using the Modified Congestion lemma [32, 36] and Partition lemma [32].

\section{Preliminaries setting}

The following two versions of the edge isoperimetric problem of a graph $G(V, E)$ have been considered in the literature [31], and are NP-complete [27].

Problem 1 (Minimum Cut Problem): Find a subset of vertices of a given graph, such that the edge cut separating this subset from its complement has minimal size among all subsets of the same cardinality. Mathematically, for a given $m$, if $\theta_{G}(m)=\min _{A \subseteq V,|A|=m}\left|\theta_{G}(A)\right|$ where $\theta_{G}(A)=\{(u, v) \in E: u \in A, v \notin$ $A\}$, then the problem is to find $A \subseteq V$ such that $|A|=m$ and $\theta_{G}(m)=\left|\theta_{G}(A)\right|$.

Problem 2 (Maximum Subgraph Problem): Find a subset of vertices of a given graph, such that the number of edges in the subgraph induced by this subset is maximal among all induced subgraphs with the same number of vertices. Mathematically, for a given $m$, if $I_{G}(m)=\max _{A \subseteq V,|A|=m}\left|I_{G}(A)\right|$ where $I_{G}(A)=\{(u, v) \in E: u, v \in A\}$, then the problem is to find $A \subseteq V$ such that $|A|=m$ and $I_{G}(m)=\left|I_{G}(A)\right|$.

For a given $m$, where $m=1,2, \ldots, n$, we consider the problem of finding a subset $A$ of vertices of $G$ such that $|A|=m$ and $\left|\theta_{G}(A)\right|=\theta_{G}(m)$. Such subsets 
are called optimal. We say that optimal subsets are nested if there exists a total order $\mathcal{O}$ on the set $V$ such that for any $m=1,2, \ldots, n$, the first $m$ vertices in this order is an optimal subset. In this case we call the order $\mathcal{O}$ an optimal order [31]. This implies that $W L\left(G, P_{n}\right)=\sum_{m=0}^{n} \theta_{G}(m)$.

Further, if a subset of vertices is optimal with respect to Problem 1, then its complement is also an optimal set. But, it is not true for Problem 2 in general. However for regular graphs a subset of vertices $S$ is optimal with respect to Problem 1 if and only if $S$ is optimal for Problem 2.

Notation: For convenience we write $E C_{f}(e)$ instead of $E C_{f}(G, H(e)$ in the sequel.

For any set $S$ of edges of $\mathrm{H}, E C_{f}(S)=\sum_{e \in S} E C_{f}(e)$.

Lemma 1. (Modified Congestion Lemma) (See [32, 36].) Let $f$ be an embedding of an arbitrary graph $G$ into $H$. Let $S$ be an edge cut of $H$ such that the removal of edges of $S$ leaves $H$ into 2 components $H_{1}$ and $H_{2}$ and let $G_{1}=f^{-1}\left(H_{1}\right)$ and $G_{2}=f^{-1}\left(H_{2}\right)$. Also $S$ satisfies the following conditions:

(i) For every edge $(a, b) \in G_{i}, i=1,2, P_{f}(a, b)$ has no edges in $S$.

(ii) For every edge $(a, b)$ in $G$ with $a \in G_{1}$ and $b \in G_{2}, P_{f}(a, b)$ has exactly one edge in $S$.

(iii) $G_{1}$ and $G_{2}$ are optimal sets.

Then $E C_{f}(S)$ is minimum and

$$
\begin{aligned}
E C_{f}(S) & =E C_{f}(S)=\sum_{v \in V\left(G_{1}\right)} \operatorname{deg}_{G}(v)-2\left|E\left(G_{1}\right)\right| \\
& =\sum_{v \in V\left(G_{2}\right)} \operatorname{deg}_{G}(v)-2\left|E\left(G_{2}\right)\right| .
\end{aligned}
$$

Lemma 2. (Partition Lemma) (See [32].) Let $f: G \rightarrow H$ be an embedding. Let $\left\{S_{1}, S_{2}, \ldots, S_{p}\right\}$ be a partition of $E(H)$ such that each $S_{i}$ is an edge cut of $H$. Then,

$$
W L_{f}(G, H)=\sum_{i=1}^{p} E C_{f}\left(S_{i}\right) .
$$

Theorem 3. (See [35].) Let $G$ be the complete $2^{p}$-partite graphs

$$
K_{2^{n-p}, 2^{n-p}, \ldots, 2^{n-p}} 1 \leq p<n .
$$


Let $H$ be a subgraph of $G$ with $|V(H)|=2^{n-1}$. Then,

$$
\left|E_{G}(H)\right| \leq 2^{2 n-p-3}\left(2^{p}-1\right) .
$$

Further, equality holds if and only if $H$ is isomorphic to a complete $2^{p}$-partite graphs $K_{2^{n-p}, 2^{n-p}, \ldots, 2^{n-p} \text {. }}$

Theorem 4. (See [35].) Let $G$ be a complete $t$-partite graph $K_{r, r, \ldots, r}$ with $n=t r$ vertices $t, r \geq 2$. Then the number of edges in a maximum subgraph on $l$ vertices of $G$ is given by

$$
\xi_{l}= \begin{cases}l(l-1) / 2 & ; l \leq t-1, \\ p^{2} t(t-1) / 2 & ; l=t, 1 \leq p \leq r, \\ p^{2} t(t-1) / 2+j p(t-1)+j(j-1) / 2 & ; \\ l=(p-1) t+j, 1 \leq j \leq t-1,2 \leq p \leq r . & \end{cases}
$$

\section{Exact wirelength of complete multipartite graphs into cycle-of-ladders}

A path of length $s$ is denoted by $P(s)$ and a cycle of length $s$ is denoted by $C(s)$. A ladder of length $s$, denoted by an $L(s)$, is a $P(s) \times K_{2}$. Each vertex of an $L(s)$ is labelled by $\left(b_{0}, b_{1}\right)$, where $b_{0}=0$ or $b_{0}=1$, and $0 \leq b_{1} \leq s$. Each edge $\left(\left(0, b_{1}\right),\left(1, b_{1}\right)\right)$ is called a rung of the ladder $L(s)$, where $0 \leq b_{1} \leq s$. Specifically, it is called the $b_{1}^{\text {th }}$ rung. The $0^{\text {th }}$ rung is called the bottom rung of the ladder. The two paths $((0,0),(0,1), \ldots,(0, s))$ and $((1,0),(1,1), \ldots,(1, s))$ are called the bands of the $L(s)$. Specifically, the former is called the $0^{\text {th }}$ band and the latter is called the $1^{\text {st }}$ band. Clearly $L(s)$ contains $2(s+1)$ verties and $3 s+1$ edges.

Definition 5. (See [33].) A cycle-of-ladders is a graph unified by a bone cycle $\mathrm{BC}$ and $k$ ladders $L D(0), L D(1), \ldots, L D(k-1)$ with $B R(0), B R(1), \ldots$, $B R(k-1)$ as the bottom rungs, respectively, such that each $\mathrm{BR}(\mathrm{i})$ is contained in the $\mathrm{BC}$ where $0 \leq i \leq k-1$.

In this section we assume that each ladder $L D(i) 0 \leq i \leq k-1$, is of length $s$. Clearly this type of cycle-of-ladders contains $2 k(s+1)$ verties and it is denoted by $C O L(k, s)$. 


\section{Embedding Algorithm}

Input: Let $G$ be the complete $2^{p}$-partite graphs $K_{2^{n-p}, 2^{n-p}, \ldots, 2^{n-p} 1 \leq p<n}$ into cycle-of-ladders $C O L(k, s)$ where $2^{n-1}=2 k(s+1)$.

Algorithm: We define a labeling of complete multipartite graphs let $V_{1}, V_{2}$, $\ldots, V_{2^{p}}$ be a partition of $G$ and label the vertices of $V_{i}, 1 \leq i \leq 2^{p}$ as follows: $j .2^{p}+i-1 \in V_{i} \mid 1 \leq i \leq 2^{p}$, when $j=2,4, \ldots, 2^{n-p}$ and $j .2^{p}+i-1 \in V_{2^{p}-i+1} \mid$ $1 \leq i \leq 2^{p}$, when $j=1,3, \ldots, 2^{n-p}$. Label the vertices of $C O L(k, s)$ as follows: Label the $0^{\text {th }}$ band vertices of $L D(0)$ from top to bottom as $1,2, \ldots, s$. For $1 \leq i \leq k-1$, label the $1^{\text {st }}$ band vertices of $L D(i)$ from bottom to top as $(2 i-1)(s+1),(2 i-1)(s+1)+1, \ldots,(2 i-1)(s+1)+s$ and the $0^{\text {th }}$ band vertices. Next from top to bottom as $(2 i-1)(s+1)+s+1,(2 i-1)(s+1)+s+$ $2, \ldots,(2 i+1)(s+1)-1$. Label the $1^{\text {st }}$ band vertices of $L D(0)$ from bottom to top as $(2 k-1)(s+1),(2 k-1)(s+1)+1, \ldots, 2 k(s+1)-1$.

Output: An embedding $f$ of complete $2^{p}$-partite graphs $K_{2^{n-p}, 2^{n-p}, \ldots, 2^{n-p}} 1 \leq p<n$ into $C O L(k, s)$ given by $f(x)=x$ with minimum wirelength.

Proof: We assume that the labels represent the vertices to which they are assigned.

For $1 \leq i \leq \frac{k}{2}$, let $A_{i}$ be the set of edges which contains all the rungs of $L D(i-1)$ and $L D\left(\frac{k}{2}+i-1\right)$. For $1 \leq j \leq \frac{k}{2}$, let $B_{j}$ be the set of edges which contains the edge between $L D(i-1)$ and $L D(i)$ and the edge between $L D\left(\frac{k}{2}+i-1\right)$ and $L D\left(\frac{k}{2}+i\right)$. For $1 \leq i \leq k, 1 \leq j \leq s$, let $S_{i}^{j}$ be the set of edges in $L D(i-1)$ which contains the edges between $(s-j+1)^{t h}$ rung and $(s-j)^{t h}$ rungs. Then $\left\{A_{i}: 1 \leq i \leq \frac{k}{2}\right\} \cup\left\{B_{j}: 1 \leq j \leq \frac{k}{2}\right\} \cup\left\{S_{i}^{j}: 1 \leq i \leq k, 1 \leq j \leq s\right\}$ is a partition of $E(C O L(k, s))$.

For each $i, 1 \leq i \leq \frac{k}{2}, E(C O L(k, s)) \backslash A_{i}$ has two components $H_{i 1}$ and $H_{i 2}$, where

$V\left(H_{i 1}\right)=\{(i-1)(2 s+2),(i-1)(2 s+2)+1, \ldots,(i-1)(2 s+2)+(k s+k-1)\}$.

Let $G_{i 1}=f^{-1}\left(H_{i 1}\right)$ and $G_{i 2}=f^{-1}\left(H_{i 2}\right)$. By Theorem $4, G_{i 1}$, is an optimal set, and each $A_{i}$ satisfies conditions $(i)$, (ii) and (iii) of the Modified Congestion Lemma. Therefore $E C_{f}\left(A_{i}\right)$ is minimum. 
For each $j, 1 \leq j \leq \frac{k}{2}, E(C O L(k, s)) \backslash B_{j}$ has two components $H_{j 1}$ and $H_{j 2}$, where

$$
\begin{aligned}
V\left(H_{j 1}\right)= & \{(2 j-1)(s+1),(2 j-1)(s+1)+1, \ldots, \\
& (2 j-1)(s+1)+(k s+k-1)\} .
\end{aligned}
$$

Let $G_{j 1}=f^{-1}\left(H_{j 1}\right)$ and $G_{j 2}=f^{-1}\left(H_{j 2}\right)$. By Theorem $4, G_{j 1}$, is an optimal set, and each $B_{j}$ satisfies conditions $(i),(i i)$ and $(i i i)$ of the Modified Congestion Lemma. Therefore $E C_{f}\left(B_{j}\right)$ is minimum.

For each $i, j, 1 \leq i \leq k, 1 \leq j \leq s, E(C O L(k, s)) \backslash S_{i}^{j}$ has two components $H_{i 1}^{j}$ and $H_{i 2}^{j}$, where

$$
V\left(H_{i 1}^{j}\right)=\left\{\begin{array}{l}
\{1, \ldots, j-1\} \cup\{2 k(s+1), \\
2 k(s+1)-2, \ldots, 2 k(s+1)-j\} \quad ; \quad \text { if } i=0, \\
\{2 i(s+1)-1,2 i(s+1)-2, \ldots, 2 i(s+1)-j\} \cup\{2 i(s+1), \\
2 i(s+1)+1, \ldots, 2 i(s+1)+j-1\} \quad ; \quad \text { if } i \neq 0 .
\end{array}\right.
$$

Let $G_{i 1}^{j}=f^{-1}\left(H_{i 1}^{j}\right)$ and $G_{i 2}^{j}=f^{-1}\left(H_{i 2}^{j}\right)$. Since $G_{i 1}^{j}$, is an optimal set, each $S_{i}^{j}$ satisfies conditions $(i),(i i)$ and $(i i i)$ of the Congestion Lemma. Therefore $E C_{f} S_{i}^{j}$ is minimum. The Partition Lemma implies that the wirelength is minimum.

Theorem 6. Let $G$ be the complete $2^{p}$-partite graphs $K_{2^{n-p}, 2^{n-p}, \ldots, 2^{n-p}} 1$ $\leq p<n$ into $C O L(k, s)$ where $2^{n-1}=2 k(s+1)$. Then the exact wirelength of $G$ into $H$ is given by

$$
W L(G, C O L(k, s))=k \cdot 2^{2 n-p-2}+k \cdot 2^{n-p}\left(2^{p}-1\right) \frac{s(s+1)}{2}-2 k \sum_{j=1}^{s} \theta_{G}(2 j) .
$$

Proof. By Modified Congestion Lemma,

i) $E C_{f}\left(A_{i}\right)=2^{2 n-p-2}\left(2^{p}-1\right), 1 \leq i \leq \frac{k}{2}$,

ii) $E C_{f}\left(B_{j}\right)=2^{2 n-p-2}\left(2^{p}-1\right), 1 \leq j \leq \frac{k}{2}$,

iii) $E C_{f}\left(S_{i}^{j}\right)=2^{n-p}\left(2^{p}-1\right) 2 j-2 \theta_{G}(2 j), 1 \leq i \leq k$ and $1 \leq j \leq s$. 
Then by Partition lemma, we have

$$
\begin{aligned}
W L(G, C O L(k, s)) & =\sum_{i=1}^{\frac{k}{2}} E C_{f}\left(A_{i}\right)+\sum_{j=1}^{\frac{k}{2}} E C_{f}\left(B_{j}\right)+\sum_{i=1}^{k} \sum_{j=1}^{s} E C_{f}\left(S_{i}^{j}\right) \\
& =\sum_{i=1}^{\frac{k}{2}} 2^{2 n-p-2}\left(2^{p}-1\right)+\sum_{j=1}^{\frac{k}{2}} 2^{2 n-p-2}\left(2^{p}-1\right) \\
& +\sum_{i=1}^{k} \sum_{j=1}^{s}\left[2^{n-p}\left(2^{p}-1\right) 2 j-2 \theta_{G}(2 j)\right] \\
& =\frac{k}{2} 2^{2 n-p-2}\left(2^{p}-1\right)+\frac{k}{2} 2^{2 n-p-2}\left(2^{p}-1\right) \\
& +k \sum_{j=1}^{s}\left[2^{n-p}\left(2^{p}-1\right) j-2 \theta_{G}(2 j)\right] \\
& =k \cdot 2^{2 n-p-2}+2 k \sum_{j=1}^{s}\left[2^{n-p}\left(2^{p}-1\right) j-2 \theta_{G}(2 j)\right] \\
& =k \cdot 2^{2 n-p-2}+2 k \sum_{j=1}^{s}\left[2^{n-p}\left(2^{p}-1\right) j-\sum_{j=1}^{s} \theta_{G}(2 j)\right] \\
& =k \cdot 2^{2 n-p-2}+2 k\left[2^{n-p}\left(2^{p}-1\right) \frac{s(s+1)}{2}-\sum_{j=1}^{s} \theta_{G}(2 j)\right. \\
& =k \cdot 2^{2 n-p-2}+k\left[2^{n-p}\left(2^{p}-1\right) s(s+1)-2 k \sum_{j=1}^{s} \theta_{G}(2 j) .\right.
\end{aligned}
$$

Hence the proof is finished.

\section{Conclusion}

In this paper, we embedded complete multipartite graphs into cycle-of-ladders to yield the minimum wirelength. Also the embedding constructed is simple and elegant, and produces exact wirelength.

Acknowledgement. This work was supported in part by the China Postdoctoral Science Foundation under Grant 2017M621579, in part by the Postdoctoral Science Foundation of Jiangsu Province under Grant 1701081B, and in part by the Project of Anhui Jianzhu University under Grant 2016QD116 and Grant 2017dc03. 


\section{References}

[1] B. Parhami, An Introduction to Parallel Processing: Algorithms and Architectures, Plenum Press, New York, 1999.

[2] V. Kianzad, S.S. Bhattacharyya, Efficient techniques for clustering and scheduling onto embedded multiprocessors, IEEE Transactions on Parallel and Distributed Systems, 17, No 7 (2006), 1847-1849.

[3] H.S. Lim, J.H. Park, K.Y. Chwa, Embedding trees in recursive circulants, Discrete Applied Mathcmatlcs, 69 (1996), 83-99.

[4] J. Opatrny, D. Sotteau, Embeddings of complete binary trees into grids and extended grids with total vertex-congestion, Discrete Applied Mathematics, 98 (2000), 237-254.

[5] S.L. Bezrukov, J.D. Chavez, L.H. Harper, M. Röttger, U.P. Schroeder, The congestion of $n$-cube layout on a rectangular grid, Discrete Mathematics, 213 (2000), 13-19.

[6] J.A. Bondy and U.S.R. Murty, Graph Theory with Applications, Macmillan, New York, 1976.

[7] J.-B. Liu, S. Wang, C. Wang, S. Hayat, Further results on computation of topological indices of certain networks, IET Control Theory Appl.; DOI: 10.1049/ietcta.2016.1237.

[8] S. Wang, B. Wei, Multiplicative Zagreb indices of $k$-trees, Discrete Appl. Math., 180 (2015), 168-175.

[9] J.-B. Liu, C. Wang, S. Wang, B. Wei, Zagreb indices and multiplicative Zagreb indices of eulerian graphs, Bull. Malays. Math. Sci. Soc.; DOI: $10.1007 / \mathrm{s} 40840-017-0463-2$.

[10] J.M. Xu, Topological Structure and Analysis of Interconnection Networks, Kluwer Academic Publishers, 2001.

[11] Y.L. Lai, K. Williams, A survey of solved problems and applications on bandwidth, edgesum, and profile of graphs, J. Graph Theory, 31 (1999), $75-94$.

[12] I. Rajasingh, J. Quadras, P. Manuel, A. William, Embedding of cycles and wheels into arbitrary trees, Networks, 44 (2004), 173-178. 
[13] A.K. Gupta, D. Nelson, H. Wang, Efficient embeddings of ternary trees into hypercubes, J. of Parallel and Distributed Computing, 63, No 6 (2003), 619-629.

[14] J. Fan, X. Jia, Embedding meshes into crossed cubes, Information Sciences, 177, No 15 (2007), 3151-3160.

[15] W.K. Chen, M.F.M. Stallmann, On embedding binary trees into hypercubes, Information Sciences, 24 (1995), 132-138.

[16] S.L. Bezrukov, Embedding complete trees into the hypercube, Discrete Applied Mathematics, 110, No 2-3 (2001), 101-119.

[17] J.F. Fang, K.C. Lai, Embedding the incomplete hypercube in books, Information Processing Letters, 96 (2005), 1-6.

[18] P.L. Lai, C.H. Tsai, Embedding of tori and grids into twisted cubes, Theoretical Computer Science, 411, No 40-42 (2010), 3763-3773.

[19] Y. Han, J. Fan, S. Zhang, J. Yang, P. Qian, Embedding meshes into locally twisted cubes, Information Sciences, 180, No 19 (2010), 3794-3805.

[20] R. Caha, V. Koubek, Optimal embeddings of generalized ladders into hypercubes, Discrete Mathematics, 233 (2001), 65-83.

[21] M. Rottger, U.P. Schroeder, Efficient embeddings of grids into grids, Discrete Applied Mathematics, 108, No 1-2 (2001), 143-173.

[22] J.D. Chavez, R. Trapp, The cyclic cutwidth of trees, Discrete Applied Mathematics, 87 (1998), 25-328.

[23] C.J. Guu, The Circular Wirelength Problem for Hypercubes, Ph.D. Dissertation, University of California, Riverside, 1997.

[24] M.C. Yang, Path embedding in star graphs, Applied Mathematics and Computation, 207, No 2 (2009), 283-291.

[25] C.H. Tsai, Embedding of meshes in M oobius cubes, Theoretical Computer Science, 401, No 1-3 (2008), 181-190.

[26] P. Manuel, Minimum average congestion of enhanced and augmented hypercube into complete binary tree, Discrete Applied Mathematics, 159, No 5 (2010), 360-366. 
[27] M.R Garey, D.S Johnson DS, Computers and Intractability, a Guide to the Theory of NP-Completeness, Freeman, San Francisco, 1979.

[28] I. Rajasingh, P. Manuel, M. Arockiaraj B. Rajan, Embeddings of circulant networks, Journal of Combinatorial Optimization, 26, No 1 (2013), 135151.

[29] P. Manuel, M. Arockiaraj, I. Rajasingh, B. Rajan, Embedding hypercubes into cylinders, snakes and caterpillars for minimizing wirelength, Discrete Applied Mathematics, 159, No 17 (2011), 2109-2116.

[30] I. Rajasingh, B. Rajan, R.S. Rajan, Embedding of special classes of circulant networks, hypercubes and generalized Petersen graphs, International Journal of Computer Mathematics, 89, No 1 (2012), 1970-1978.

[31] S.L. Bezrukov, S.K. Das, R. Elsässer, An edge-isoperimetric problem for powers of the Petersen graph, Annals of Combinatorics, 4 (2000), 153-169.

[32] P. Manuel, I.Rajasingh, B.Rajan, H. Mercy, Exact wirelength of hypercube on a grid, Discrete Appl. Math., 157, No 7 (2009), 1486-1495.

[33] J.F. Fang, The bipancycle-connectivity of the hypercube, Information Sciences, 178 (2008), 4679-4687.

[34] S.K. Vaidya, S. Srivastav, V.J. Kaneria, G.V. Ghodasara, Cordial and 3-equitable labeling of star of a cycle, Discrete Mathematics, 24 (2008), 54-64.

[35] R.S. Rajan, T.M Rajalaxmi, Jia-Baco, G. Sethuraman, Wirelength of embedding complete multipartite graphs into certain graphs, Manuscript.

[36] M. Miller, R.S. Rajan, N. Parthiban, I. Rajasingh, Minimimum linear arrangment of incomlete hypercubes, The Computer Journal, No 2 (2015), 331-337. 
